\title{
Interactions among pressure, density, vorticity and their gradients in compressible turbulent channel flows
}

\author{
LIANG WEI AND ANDREW POLLARD \\ Department of Mechanical and Materials Engineering, Queen's University, Kingston, \\ ON K7L 3N6, Canada
}

(Received 17 March 2010; revised 25 November 2010; accepted 26 November 2010; first published online 14 February 2011)

The interactions among pressure, density, vorticity and their gradients in compressible turbulent channel flows (TCF) are studied using direct numerical simulations (DNS). DNS of three isothermal-wall TCF for Mach number $M a=0.2,0.7$, and 1.5, respectively are performed using a discontinuous Galerkin method (DGM). The Reynolds numbers of these three cases are $\approx 2800$, based on the bulk velocity, bulk density, half channel width and dynamic viscosity at the wall. A high cross-correlation between density and spanwise vorticity occurs at $y^{+} \approx 4$, which is coincident with the peak mean spanwise baroclinicity. The relationship between the spanwise baroclinicity and the correlation is analysed. The difference between the evolution of density and spanwise vorticity very near the wall is discussed. The transport equation for the mean product of density and vorticity fluctuations $\left\langle\rho^{\prime} \omega_{i}^{\prime}\right\rangle$ is presented and the distributions of terms in the $\left\langle\rho^{\prime} \omega_{z}^{\prime}\right\rangle$ transport equation indicate that the minima and maxima of the profiles are located around $y^{+} \approx 5$. The connection between pressure gradients and vorticity fluxes for compressible turbulent flows with variable viscosity has been formulated and verified. High correlations (0.7-1.0) between pressure gradient and vorticity flux are found very close to the wall $\left(y^{+}<5\right)$. The correlation coefficients are significantly influenced by $M a$ and viscosity in this region. Turbulence advection plays an important role in destroying the high correlations between pressure gradient and vorticity flux in the region away from the wall $\left(y^{+}>5\right)$.

Key words: compressible boundary layers, turbulent boundary layers, vortex dynamics

\section{Introduction}

Vorticity can be generated at a solid surface, and, in the case of variable density flows through the cross-product of density gradient and pressure gradient, which is referred to as baroclinic torque or baroclinicity. Previous studies on vorticity dynamics include the linkage between pressure gradient and vorticity generation on a solid wall in laminar and turbulent flows; however, few studies exist for the pressure, density and vorticity interactions, as well as quantitatively evaluating the linkage between pressure gradient and vorticity generation as a function of Mach number and wallnormal distance. Clarifying the interactions among pressure, density, vorticity and 
their gradients is of great importance to understand the mechanism of vorticity evolution in wall-bounded compressible turbulent flows.

Viscous vorticity flux is often used to describe vorticity generation at a solid surface (Andreopoulos \& Agui 1996). It was defined by Lighthill (1963) for a two-dimensional incompressible laminar boundary layer: $\sigma=-(\nu \partial \omega / \partial y)_{w}$, where $\omega$ is the vorticity, $v$ is the kinematic viscosity and subscript $w$ denotes on the wall. Panton (1984) extended the definition to three-dimensional incompressible flow: $\sigma=-(v \boldsymbol{n} \cdot \nabla \boldsymbol{\omega})_{w}$, where $\boldsymbol{n}$ is the normal vector to the solid surface and $\omega$ is the vorticity vector. $\mathrm{Wu}, \mathrm{Wu} \& \mathrm{Wu}$ (1988) proposed a definition for compressible flow: $\sigma=-\boldsymbol{n} \cdot \nabla(\mu \omega)$, where $\mu$ is the dynamic viscosity. The current study considers viscous vorticity flux both on the wall and the regions away from the wall and the vorticity flux ('viscous' will be omitted for simplicity hereafter) is not limited to the wall-normal direction, and therefore a vorticity flux tensor is proposed: $\sigma_{i j}=\partial \mu \omega_{i} / \partial x_{j}$.

The influence of pressure gradients and fluid properties on vorticity flux has been considered by a number of researchers. Lighthill (1963) proposed that the vorticity flux (vorticity source strength) was dominated by wall-tangential pressure gradients in a two-dimensional laminar boundary layer. Wu et al. (1988) extended the relationship between vorticity and pressure to a viscous compressible flow under the assumption of constant viscosity. A general theory for the interaction between vorticity-dilatation field and the solid surface was outlined. Gad-El-Hak (1990) found that vorticity flux can be affected by the wall-normal gradient of the kinematic viscosity as a result of surface heating and chemical reaction. Wu, Wu \& Wu (1993) considered vorticity generation on an oscillating wall and found that wall oscillation generated additional vorticity flux, which could affect either positively or negatively an existing vorticity field. They claimed that this effect was partially responsible for the mechanism of vortex flow control by waves. Developments of boundary vorticity dynamics theory were reviewed by $\mathrm{Wu} \& \mathrm{Wu}(1998)$.

The interaction between pressure gradients and vorticity flux was employed in flow control. Koumoutsakos (1999) proposed a control algorithm based on the measurement and manipulation of the wall vorticity flux (or, equivalently, pressure gradient) and applied this algorithm to a low-Reynolds-number turbulent incompressible channel flow. The simulation showed that the resulting skin friction drag reduction could be of the order of $40 \%$. Lee \& Kim (2002) also investigated vorticity generation and found it significant in the viscous sublayer of a turbulent boundary layer. Drag reduction could be achieved through a suppression of spanwise disturbances in the sublayer, which caused suppression of near-wall turbulence structures.

The previous studies have considered the interplay between pressure gradient and vorticity flux applied to incompressible flow; the relationship between them has been considered neither for compressible flow as a function of distance from a solid wall nor for the case when the viscosity is spatially variable. The motivation for the current study is therefore to explore the variation in the correlations of the pressure gradients and vorticity flux in near-wall turbulent flows as a function of Mach number for a constant wall temperature, and to study the interactions among pressure, density and vorticity in compressible wall-bounded turbulent flows.

The paper is organized as follows. The next section presents the formulation of the linkage between pressure gradient and vorticity flux for compressible flows with variable viscosity. It is then followed by the analysis of the data from the direct numerical simulation (DNS) of compressible turbulent wall-bounded flows at different Mach numbers, in which cross-correlations are proposed to quantitatively evaluate the linkage between pressure, density and vorticity and the linkage between 
pressure gradient and vorticity flux. The budget for density vorticity correlation is obtained and analysed. The influence of Mach number, viscosity and advection on the correlations as well as the related flow physics is also discussed. The final section provides a summary of the current work.

\section{Vorticity dynamics formulation for compressible turbulent flows with variable viscosity}

Consider continuity, momentum and energy equations with an added driving force for compressible, variable property flow:

$$
\begin{gathered}
\frac{\partial \rho}{\partial t}+\frac{\partial \rho u_{j}}{\partial x_{j}}=0, \\
\frac{\partial \rho u_{i}}{\partial t}+\frac{\partial\left(\rho u_{i} u_{j}\right)}{\partial x_{j}}+\frac{\partial p}{\partial x_{i}}=\frac{\partial \tau_{i j}}{\partial x_{j}}+\rho f_{i}, \\
\frac{\partial E}{\partial t}+\frac{\partial(E+p) u_{j}}{\partial x_{j}}=\frac{\partial\left(\tau_{i j} u_{i}-q_{j}\right)}{\partial x_{j}}+\rho f_{i} u_{i},
\end{gathered}
$$

where $\tau_{i j}$ is the viscous stress tensor: $\tau_{i j}=\mu\left(\partial u_{i} / \partial x_{j}+\partial u_{j} / \partial x_{i}\right)-(2 \mu / 3) \delta_{i j} \partial u_{k} / \partial x_{k}$, $\delta_{i j}$ is Kronecker's delta and the dynamic viscosity $\mu$ is a function of temperature: $\mu=\mu(T)$, based on Sutherland's theory of viscosity (Schlichting 1979); $f_{i}$ is the driving force; $E$ is the total energy, defined as $E=p /(\gamma-1)+0.5 \rho u_{i} u_{i}$, where $\gamma$ is the specific heat ratio and $q_{j}$ is the heat flux: $q_{j}=-\kappa \partial T / \partial x_{j}$, where $\kappa$ is the thermal conductivity. The equation of state for a perfect gas is: $p=\rho R T$, where $R$ is the gas constant.

The momentum equation (2.2) can be rewritten as (no assumption has been made about the spatial variation of viscosity):

$$
\rho \frac{\partial u_{i}}{\partial t}+\rho u_{j} \frac{\partial u_{i}}{\partial x_{j}}+\frac{\partial p}{\partial x_{i}}=-\mu \varepsilon_{i j k} \frac{\partial \omega_{k}}{\partial x_{j}}+\frac{4}{3} \mu \frac{\partial \Theta}{\partial x_{i}}+\frac{\tau_{i j}}{\mu} \frac{\partial \mu}{\partial x_{j}}+\rho f_{i},
$$

where $\varepsilon_{i j k}$ is the permutation symbol, $\omega_{k}$ is the vorticity $\omega_{k}=\varepsilon_{k l m} \partial u_{m} / \partial x_{l}$ and $\Theta$ denotes the dilatation $\Theta=\partial u_{j} / \partial x_{j}$.

The relationship between pressure gradient and vorticity gradient is affected by the following terms:

(i) the substantial derivative: $\rho \partial u_{i} / \partial t+\rho u_{j} \partial u_{i} / \partial x_{j}$, of which $\rho \partial u_{i} / \partial t$ is the unsteady term and $\rho u_{j} \partial u_{i} / \partial x_{j}$ is the advection term.

(ii) the vorticity gradient term: $-\mu \varepsilon_{i j k} \partial \omega_{k} / \partial x_{j}$, which involves vorticity flux.

(iii) the dilatation gradient term: $(4 \mu / 3) \partial \Theta / \partial x_{i}$, which is related to compressibility effects.

(iv) the viscosity gradient term: $\left(\tau_{i j} / \mu\right) \partial \mu / \partial x_{j}$, which denotes the effect of variations in viscosity mainly caused by the variations in temperatures (or heat flux).

(v) the driving force term: $\rho f_{i}$.

Simplifications to (2.4) can be made on a no-slip isothermal wall $\left(y=0\right.$ or $\left.x_{2}=0\right)$, where velocities are zero $u, v, w=0$ and temperature constant $T=$ constant. First and higher order derivatives of velocities and viscosity with respect to $t, x$ and $z$ (or $x_{1}$ and $x_{3}$ ) are also zero on the wall. The driving forces in the current simulations are: $f_{x}=$ constant, $f_{y}=f_{z}=0$. It then follows, on the wall, that

$$
\frac{\partial p}{\partial x}=-\mu \frac{\partial \omega_{z}}{\partial y}+\frac{\partial u}{\partial y} \frac{\partial \mu}{\partial y}+\frac{4}{3} \mu \frac{\partial \Theta}{\partial x}+\rho f_{x},
$$




$$
\begin{gathered}
\frac{\partial p}{\partial y}=-\mu \frac{\partial \omega_{x}}{\partial z}+\mu \frac{\partial \omega_{z}}{\partial x}+\frac{4}{3} \mu \frac{\partial \Theta}{\partial y}+\frac{4}{3} \Theta \frac{\partial \mu}{\partial y}, \\
\frac{\partial p}{\partial z}=\mu \frac{\partial \omega_{x}}{\partial y}+\frac{\partial w}{\partial y} \frac{\partial \mu}{\partial y}+\frac{4}{3} \mu \frac{\partial \Theta}{\partial z} .
\end{gathered}
$$

Further refinement of (2.5)-(2.7) gives,

$$
\begin{gathered}
\frac{\partial p}{\partial x}=-\frac{\partial \mu \omega_{z}}{\partial y}+\frac{4}{3} \frac{\partial \mu \Theta}{\partial x}+\rho f_{x}, \\
\frac{\partial p}{\partial y}=-\frac{\partial \mu \omega_{x}}{\partial z}+\frac{\partial \mu \omega_{z}}{\partial x}+\frac{4}{3} \frac{\partial \mu \Theta}{\partial y}, \\
\frac{\partial p}{\partial z}=\frac{\partial \mu \omega_{x}}{\partial y}+\frac{4}{3} \frac{\partial \mu \Theta}{\partial z} .
\end{gathered}
$$

Note that the terms in the above equations are instantaneous quantities although some terms may have zero mean when averaged over time and the homogeneous directions. It can be inferred from the above equations that the vorticity generation from the wall is affected by pressure gradients, the driving force, dilatation gradients (compressibility effects) and viscosity gradients. Specifically, the vorticity flux in the wall-normal direction may be determined by wall-tangential pressure gradients/driving force, wall-tangential dilatation gradients and wall-normal viscosity gradients. On the other hand, the vorticity flux in the wall-tangential directions may be determined by the wall-normal pressure gradient, driving force, wall-normal dilatation gradients and viscosity gradients. It should be noted that when the region away from the wall is considered, the vorticity flux becomes more complicated and advection probably plays an increasingly important role (see (2.4)).

\section{DNS of compressible turbulent channel flow}

DNS of fully developed compressible turbulent flow between two isothermal parallel plates at three different Mach numbers was carried out using a discontinuous Galerkin method (DGM). DGM is a finite-element-based method that uses numerical fluxes on element boundaries, which draws from finite volume method, so that it can accommodate discontinuous solutions on element boundaries. It has many attractive features including: high order accuracy, highly parallelizable and well suitable for complex geometries (see Karniadakis \& Sherwin 2005 and Cockburn, Karniadakis \& Shu 2000).

The Mach numbers of the cases considered were $M a=0.2, M a=0.7$ and $M a=1.5$, respectively referred to as $\mathrm{Ma} 02, \mathrm{Ma} 07$ and Ma15 hereafter. The Mach number is defined as $M a=U_{m} / \sqrt{\gamma R T_{w}}$, where $U_{m}$ is the mean bulk velocity and $T_{w}$ the wall temperature $\left(T_{w}=293.15 \mathrm{~K}\right.$ for all three cases). Note that the local Mach number $M a(y)$ is defined as the local mean velocity divided by the local mean sound speed. For the case Ma15, the sonic line $(M a(y)=1)$ is located at $y=0.115 h$ ( $h$ is the channel half-width) from the wall (see Wei 2009). The non-dimensional heat flux, $B_{q}=q_{w} /\left(\rho_{w} c_{p} u_{\tau} T_{w}\right)$, where the subscript $w$ denotes the wall, $c_{p}$ is the specific heat capacity at constant pressure and $u_{\tau}$ the friction velocity is $-0.001,-0.012$ and -0.047 , for cases Ma02, Ma07 and Ma15, respectively.

The Reynolds number was $\approx 2800$ in all cases, based on $U_{m}, h$, the mean bulk density $\rho_{m}$ and the dynamic viscosity at the wall $\mu_{w}$. The computational domain and coordinate system was $12 h, 2 h$ and $6 h$ in the streamwise, wall-normal and spanwise 


\begin{tabular}{lccccccccccc}
\hline Case & $M a$ & $R e$ & $R e_{\tau}$ & $\operatorname{Pr}$ & $\gamma$ & $L_{x}$ & $L_{y}$ & $L_{z}$ & $\Delta x^{+}$ & $\Delta y_{\min }^{+} / \Delta y_{\max }^{+}$ & $\Delta z^{+}$ \\
MKM & 0 & 2800 & 178 & $/$ & $/$ & $4 \pi h$ & $2 h$ & $\frac{4}{3} \pi h$ & 17.7 & $0.1 / 4.4$ & 5.9 \\
CKM & 1.5 & 3000 & 222 & 0.7 & 1.4 & $4 \pi h$ & $2 h$ & $\frac{4}{3} \pi h$ & 19 & $0.1 / 5.9$ & 12 \\
Ma02 & 0.2 & 2772 & 180 & 0.72 & 1.4 & $12 h$ & $2 h$ & $6 h$ & 4.74 & $0.19 / 2.81$ & 4.74 \\
Ma07 & 0.7 & 2795 & 186 & 0.72 & 1.4 & $12 h$ & $2 h$ & $6 h$ & 4.89 & $0.19 / 2.89$ & 4.89 \\
Ma15 & 1.5 & 2811 & 208 & 0.72 & 1.4 & $12 h$ & $2 h$ & $6 h$ & 5.42 & $0.22 / 3.24$ & 5.42
\end{tabular}

TABLE 1. Physical and numerical parameters.

directions, respectively. The number of grid elements was $24 \times 15 \times 12$, in these same directions $(x, y, z)$, respectively. The distribution of grid elements in $x$ and $z$ directions was uniform. A hyperbolic tangent function was used to distribute elements in the $y$ direction. A DGM of 10th-order polynomial $(P=10)$ and a second-order AdamsBashforth time scheme were employed. Over-integration with $2 P$ grid points per direction was applied to avoid aliasing errors. Details of the method can be found in Wei (2009) and Wei \& Pollard (2010). Physical and numerical parameters of the current simulations are compared with Moser, Kim \& Mansour (1999) (MKM) and Coleman, Kim \& Moser (1995) (CKM) in table 1.

The flow was assumed to be periodic in the streamwise and spanwise directions. An analysis of the Kolmogorov microscale, one-dimensional energy spectra and correlations showed that the grid resolution was fine enough to capture the smallest scales and the domain size was large enough to include the largest eddies in the flow (see Wei 2009).

The current simulation results that include mean profiles, second-order and higherorder statistics were compared with the cases MKM and CKM (see Wei 2009 and Wei \& Pollard 2010). Very good agreement was found between the current simulations and the two reference cases.

\subsection{Cross-correlations between pressure, density and vorticity}

The vorticity equation for compressible flow can be written as

$$
\frac{\partial \omega_{i}}{\partial t}+u_{j} \frac{\partial \omega_{i}}{\partial x_{j}}=\omega_{j} \frac{\partial u_{i}}{\partial x_{j}}-\omega_{i} \frac{\partial u_{j}}{\partial x_{j}}+\beta_{i}+\eta_{i},
$$

where $\beta_{i}$ is the baroclinic vector or baroclinicity, which represents a source for vorticity generation (it is a measure of the alignment of density and pressure isosurfaces, and is non-zero for compressible flows),

$$
\beta_{i}=\epsilon_{i j k} \frac{1}{\rho^{2}} \frac{\partial \rho}{\partial x_{j}} \frac{\partial p}{\partial x_{k}},
$$

and $\eta_{i}$ is the shear stress term, which encompasses viscous effects,

$$
\eta_{i}=\epsilon_{i j k} \frac{\partial}{\partial x_{j}}\left(\frac{1}{\rho} \frac{\partial \tau_{k m}}{\partial x_{m}}\right) .
$$

The interaction between pressure, density and vorticity is explored using crosscorrelations. The cross-correlation coefficient (between variable $a$ and variable $b$ ) is defined as:

$$
R_{a: b}=\frac{\left\langle a^{\prime} b^{\prime}\right\rangle}{\left\langle a^{\prime} a^{\prime}\right\rangle^{0.5}\left\langle b^{\prime} b^{\prime}\right\rangle^{0.5}},
$$




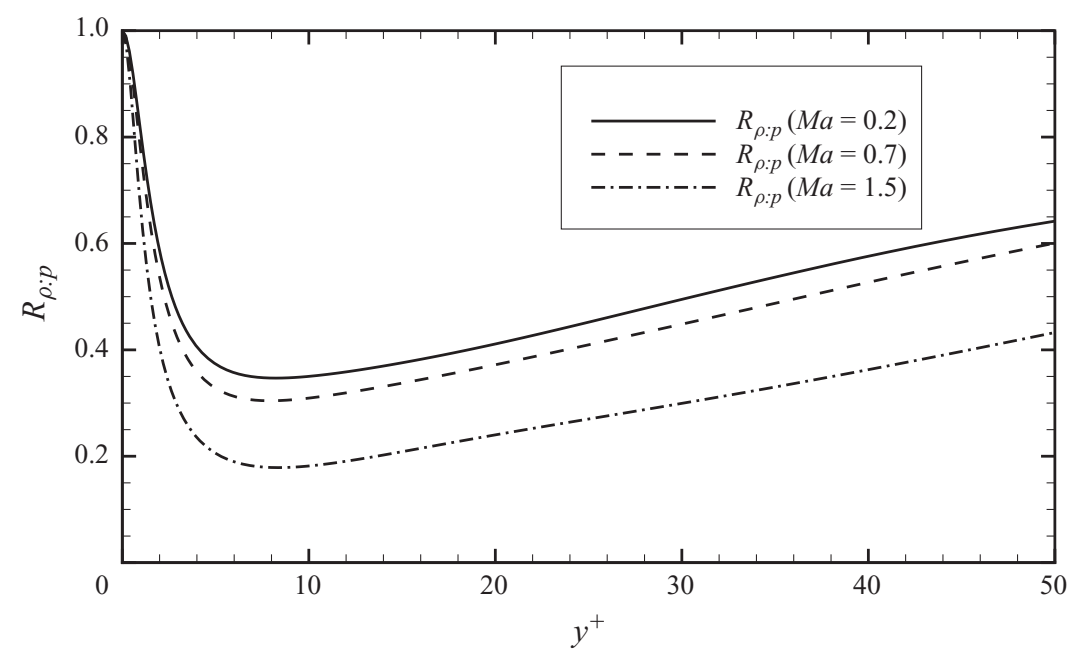

FIGURE 1. Cross-correlation coefficients between pressure and density near the wall.

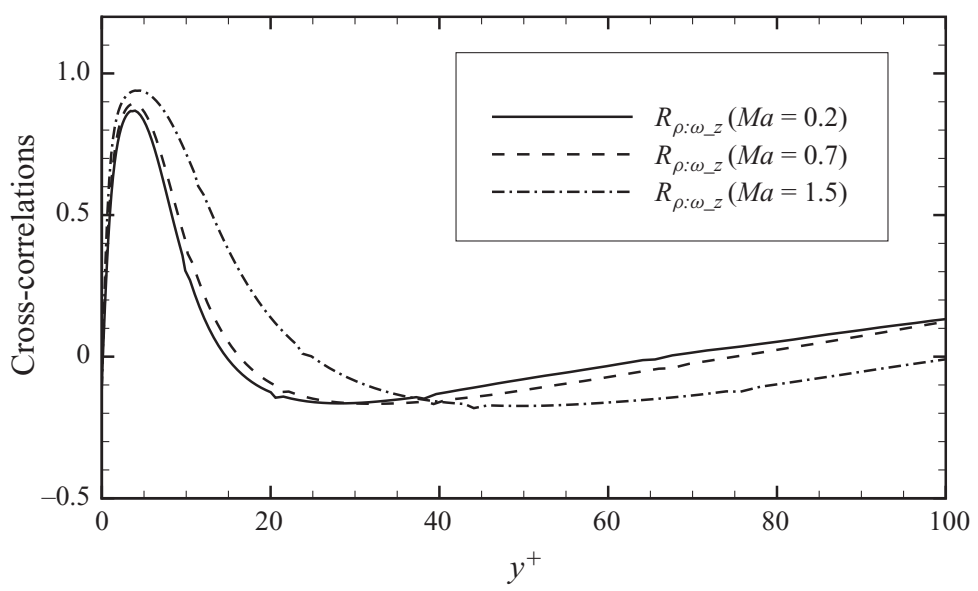

FIGURE 2. Cross-correlation coefficients between density $(\rho)$ and spanwise vorticity $\left(\omega_{-} z\right)$ close to the wall in wall units.

where angle brackets $(\langle\rangle)$ denote average over time and $x-z$ directions and the apostrophe (') denotes fluctuations with respect to the mean.

For an isothermal wall, pressure and density should be perfectly correlated on the wall through the equation of state as confirmed in figure 1, since the temperature fluctuations are zero on the isothermal wall. The cross-correlation coefficient quickly drops to a minimum value at the region $y^{+} \approx 8$ for the case Ma15, which roughly corresponds to the maximum temperature fluctuations (see the temperature fluctuation figure shown later in this section). The correlation between density and pressure very close to the wall decreases with increasing Mach number, which is due to the increase of the temperature fluctuations with Mach number.

The correlation between pressure and every component of the vorticity is found to be negligible; however, there is a significant correlation between density and spanwise vorticity very close to the (bottom) wall for all three cases Ma02, Ma07 and Ma15 (see figure 2). Note that the correlations near the top and bottom walls have the same 

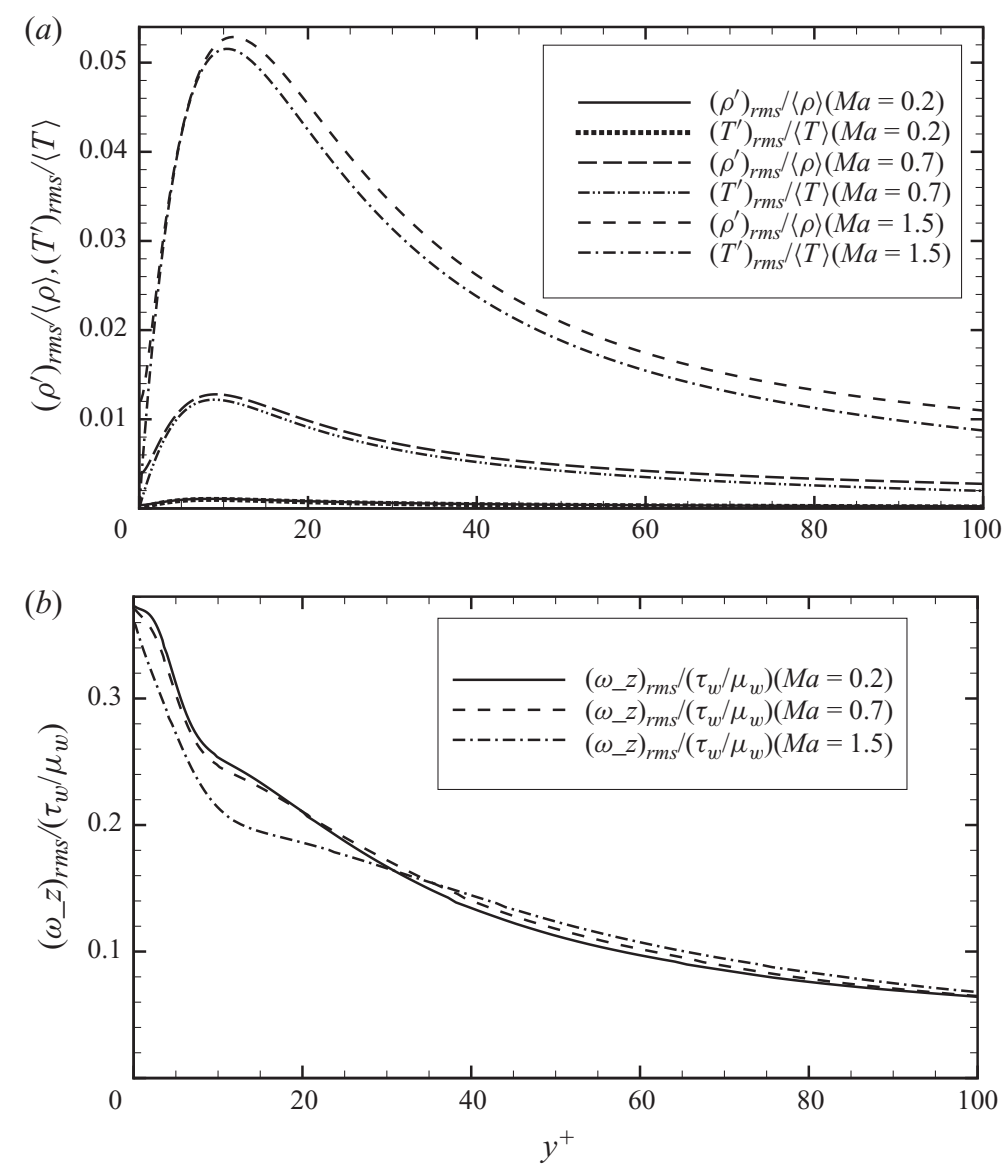

Figure 3. Profiles of density, temperature and spanwise vorticity fluctuations: $(a)\left(\rho^{\prime}\right)_{r m s} /\langle\rho\rangle$, and $\left(T^{\prime}\right)_{r m s} /\langle T\rangle ;(b)\left(\omega_{z}^{\prime}\right)_{r m s} /\left(\tau_{w} / \mu_{w}\right)$, where $\tau_{w}$ is the shear stress at the wall.

magnitude but are opposite in sign. The cross-correlations in this figure indicate that the maximum correlation occurs around $y^{+} \approx 4$ for all cases (there is a very slight shift away from the wall with increasing Mach number), an observation not previously reported in the literature. The peak correlation coefficients are around 0.9 and they increase (mildly) with Mach number. In other words, compressible isothermal-wall channel flows share a common feature that density and spanwise vorticity are highly correlated at $y^{+} \approx 4$. This will be further explored shortly. It would be interesting to determine if other compressible isothermal-wall-bounded flows, such as pipe flows, have similar regions.

Fluctuations of spanwise vorticity and thermodynamic properties such as density and temperature are also investigated to demonstrate the dynamical significance of the correlation between density and spanwise vorticity. The root-mean-square (RMS) of density $\rho$, temperature $T$ and spanwise vorticity $\omega_{z}\left(\omega_{-} z\right.$ or $\left.\omega_{3}\right)$ is shown in figure 3 . Figure 3(a) indicates that RMS density $\left(\rho^{\prime}\right)_{r m s} /\langle\rho\rangle$ and temperature $\left(T^{\prime}\right)_{r m s} /\langle T\rangle$ increase significantly and the location of the peaks of the profiles shifts slightly away from the wall as Mach number increases. The difference between the profiles of $\left(\rho^{\prime}\right)_{r m s} /\langle\rho\rangle$ and $\left(T^{\prime}\right)_{r m s} /\langle T\rangle$ at each Mach number is small over most regions except on the wall, where the temperature fluctuations are zero. The difference between these 
(a)

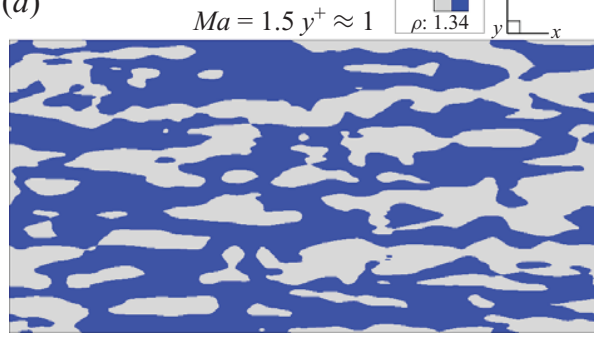

(b)

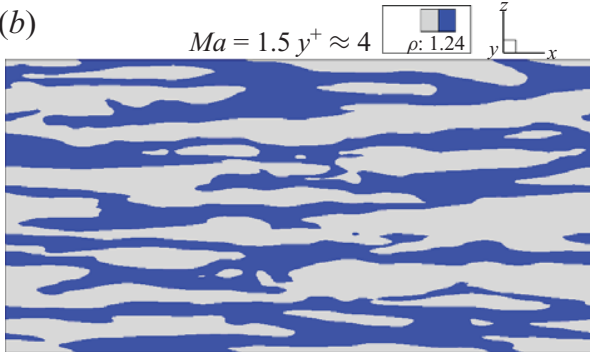

(c)

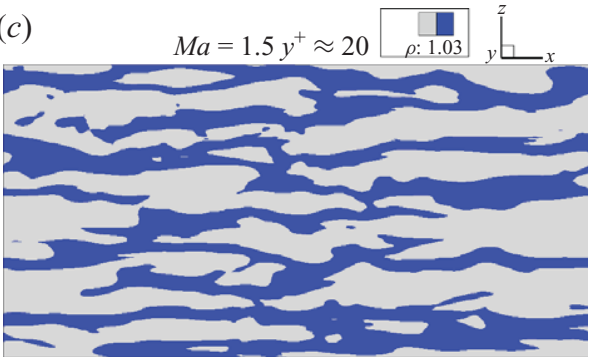

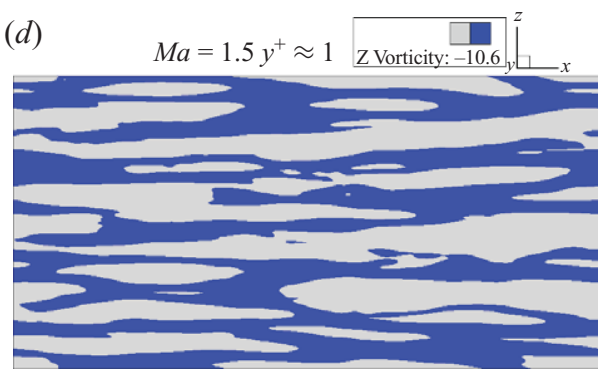

(e)

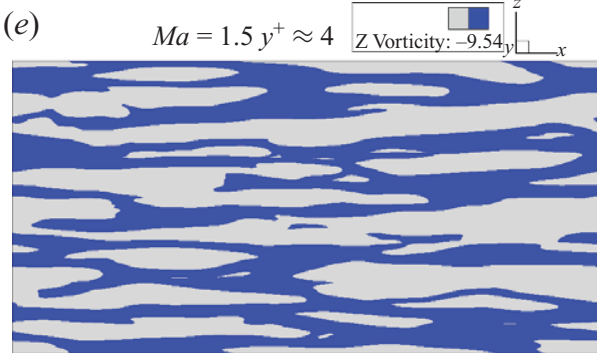

$(f)$ $M a=1.5 y^{+} \approx 20$ Z Vorticity: $-2.99, y$

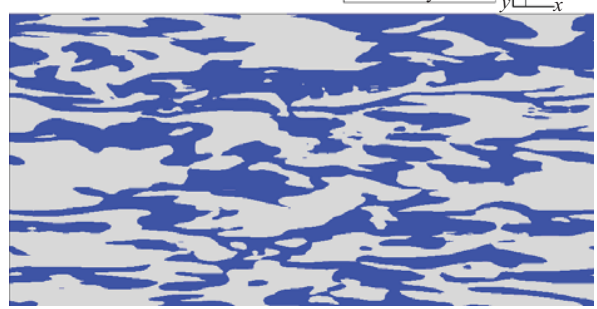

Figure 4. (Colour online) Contours of density and spanwise vorticity, nondimensionalized by $\rho_{m}$ and $U_{m} / h$, respectively, on $(x-z)$ planes close to the wall for the case Ma15. (a) Density at $y^{+} \approx 1 ;(b)$ Density at $y^{+} \approx 4 ;(c)$ Density at $y^{+} \approx 20 ;(d)$ Spanwise vorticity at $y^{+} \approx 1 ;(e)$ Spanwise vorticity at $y^{+} \approx 4 ;(f)$ Spanwise vorticity at $y^{+} \approx 20$. Threshold is taken as the mean value at the respective $y$ location so that the blue colour represents the positive fluctuations with respect to the mean and the grey colour negative.

two profiles seems to increase marginally with increasing Mach number. The profiles of spanwise vorticity fluctuations shown in figure $3(b)$ change slightly with Mach number except for a small region around $y^{+} \approx 15$, where the maximum turbulence production occurs. It can be seen from both figures that fluctuations of density and spanwise vorticity are significant in the region around $y^{+}=4$, especially when the Mach number is greater than one.

To explore the spatial distribution of density and spanwise vorticity around $y^{+}=4$, snapshots of density and spanwise vorticity contours at $y^{+} \approx 1,4,20$ for the case Ma15 are given in figure 4. In the figure, the blue and grey shades represent positive and negative fluctuations, respectively about a mean value; The mean value is noted in the graph legend. At $y^{+} \approx 4$, the density (figure $4 b$ ), and the spanwise vorticity (figure $4 e$ ) share similar long regions of streamwise 'streaks' in both size and position, which further confirms their high correlation around this location ('streaks' is used 
here to convey the idea of contiguous regions and hereafter, the quotation marks are not used). However, the density and spanwise vorticity behave differently at $y^{+} \approx 1$ and $y^{+} \approx 20$. The spanwise vorticity streaks at $y^{+} \approx 1$ (figure $4 d$ ) look very similar with those at $y^{+} \approx 4$ (figure $4 e$ ), which is different in shape and size from those streaks at $y^{+} \approx 20$ (figure $4 f$ ). The spanwise vorticity is defined as $\omega_{z}=-\partial u / \partial y+\partial v / \partial x$, where $-\partial u / \partial y$ is the dominant term and $\partial v / \partial x$ is negligible within the viscous sublayer $y^{+}<5$. The streaks of the spanwise vorticity should be similar as those of the streamwise velocity in this viscosity-dominant region. Away from the wall, the spanwise vorticity streaks become less coherent and organized with decreasing viscous effects and increasing turbulence activity.

The development of the density is different. Unlike the spanwise vorticity, the density streaks change considerably within the viscous sublayer, but the change between $y^{+} \approx 4$ and $y^{+} \approx 20$ is small (see figure $4 a-c$ ). The main reason is that temperature has a significant influence on density through the equation of state including the influence of the isothermal wall. On the isothermal wall, the temperature fluctuations are zero and density and pressure are non-zero and are perfectly correlated. The influence of temperature fluctuations on the density streaks is negligible. Away from the wall, temperature fluctuations increase in magnitude and play an increasingly important role on the density fluctuations. Note that temperature streaks are coherent close to the wall, as shown in figure 5. The streamwise coherence of density streaks is increased from $y^{+} \approx 1$ to $y^{+} \approx 4$. Although the spatial contiguousness in density and spanwise vorticity develop differently with distance from the wall, they are well correlated at $y^{+} \approx 4$, as noted in figure 2 .

The mean spanwise component of the baroclinic vector $\left(\left\langle\beta_{3}\right\rangle\right.$ or $\left.\left\langle\beta_{-} z\right\rangle\right)$, nondimensionalized by $U_{m}^{2} / h^{2}$, for three cases is displayed in figure 6 . Note that the profiles for the cases $\mathrm{Ma} 02$ and $\mathrm{Ma07}$ are almost identical. The locations of the peak values are the same as the correlation in figure 2, although the magnitude of the peak for Ma15 is markedly higher than the others. The trend of the curves is similar and the intersection between the profile for the case Ma15 and those for the cases $\mathrm{Ma} 02 / \mathrm{Ma} 07$ is located approximately at $y^{+} \approx 40$ in both figures. A question arises: is the correlation between the density and spanwise vorticity and the mean spanwise baroclinicity related?

The near-wall relationship between the mean spanwise baroclinic torque and the correlation between density and spanwise vorticity can be obtained through rearranging the vorticity equation (3.1) to obtain $\left\langle\beta_{3}\right\rangle$ as a function of $\left\langle\rho^{\prime} \omega_{3}^{\prime}\right\rangle$. The unsteady terms are removed when taking average over time and $x-z$ directions of (3.1). Since $\eta_{i}$ in (3.1) is the only term that contains both density and vorticity, nonlinear terms can be considered as a source term. Let $i=3$, then (3.1) can be rewritten as

$$
\left\langle\beta_{3}\right\rangle=\frac{\partial}{\partial x_{2}}\left\langle\frac{1}{\rho} \frac{\partial \mu \omega_{3}}{\partial x_{2}}\right\rangle+S_{1},
$$

where $S_{1}$ is a source term which is a combination of the terms that do not have both $\rho$ and $\omega_{3}$. Further expansion of (3.5) gives

$$
\left\langle\beta_{3}\right\rangle=-\frac{\partial}{\partial x_{2}}\left(\frac{\langle\mu\rangle}{\langle\rho\rangle} \frac{\partial\left\langle\rho^{\prime} \omega_{3}^{\prime}\right\rangle}{\partial x_{2}}\right)-\frac{\partial}{\partial x_{2}}\left(\frac{\left\langle\rho^{\prime} \omega_{3}^{\prime}\right\rangle}{\langle\rho\rangle^{2}} \frac{\partial\langle\mu\rangle}{\partial x_{2}}\right)+S_{2}
$$

where $S_{2}$ is a sum of the terms that do not contain $\left\langle\rho^{\prime} \omega_{3}^{\prime}\right\rangle$. 

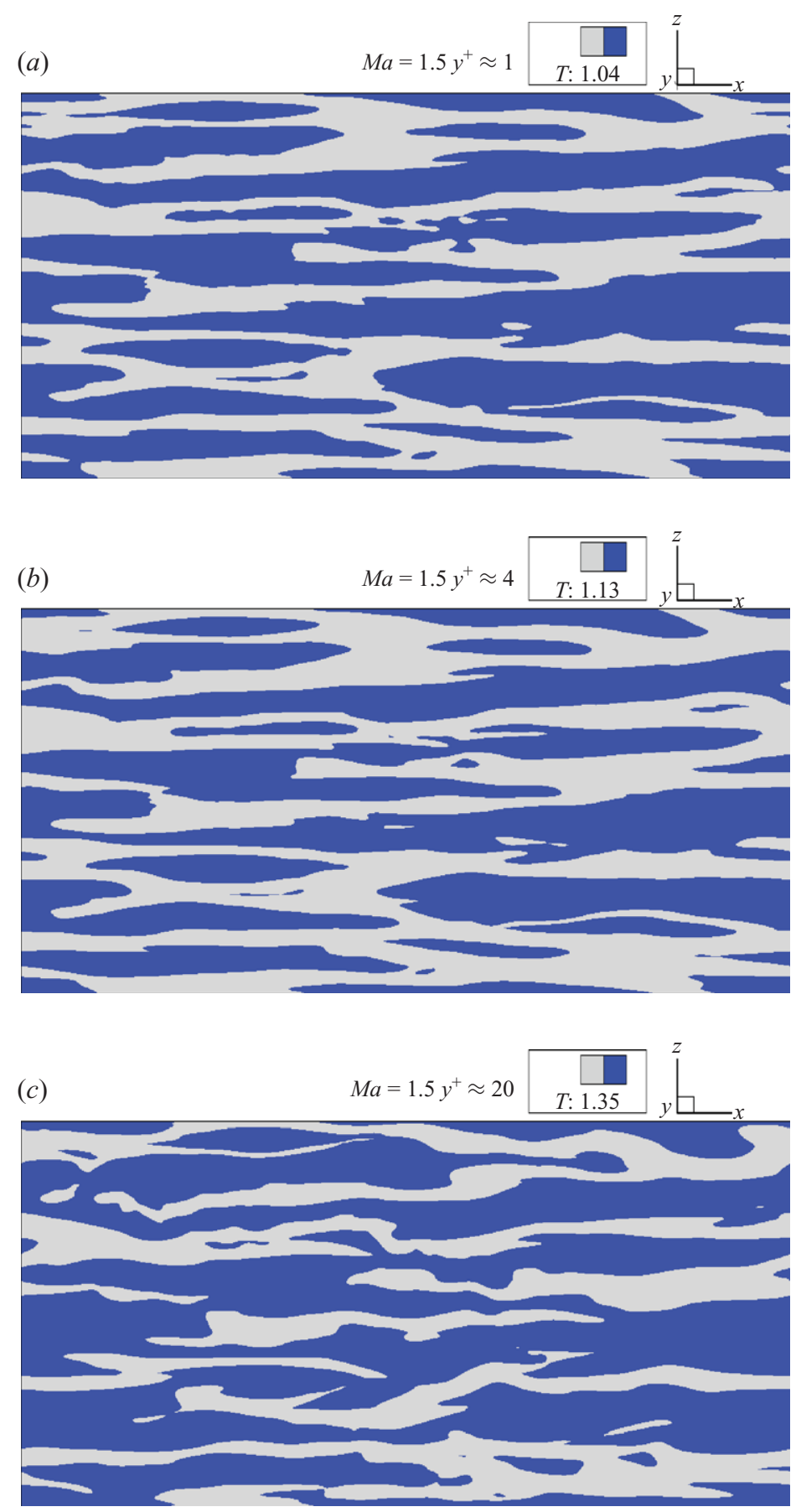

FIGURE 5. (Colour online) Contours of temperature, nondimensionalized by the wall temperature $T_{w}$, on $(x-z)$ planes close to the wall for the case Ma15. (a) $y^{+} \approx 1 ;(b) y^{+} \approx 4$; (c) $y^{+} \approx 20$. Threshold is taken as the mean value at the respective $y$ location so that the blue colour represents the positive fluctuations with respect to the mean and the grey colour negative. 


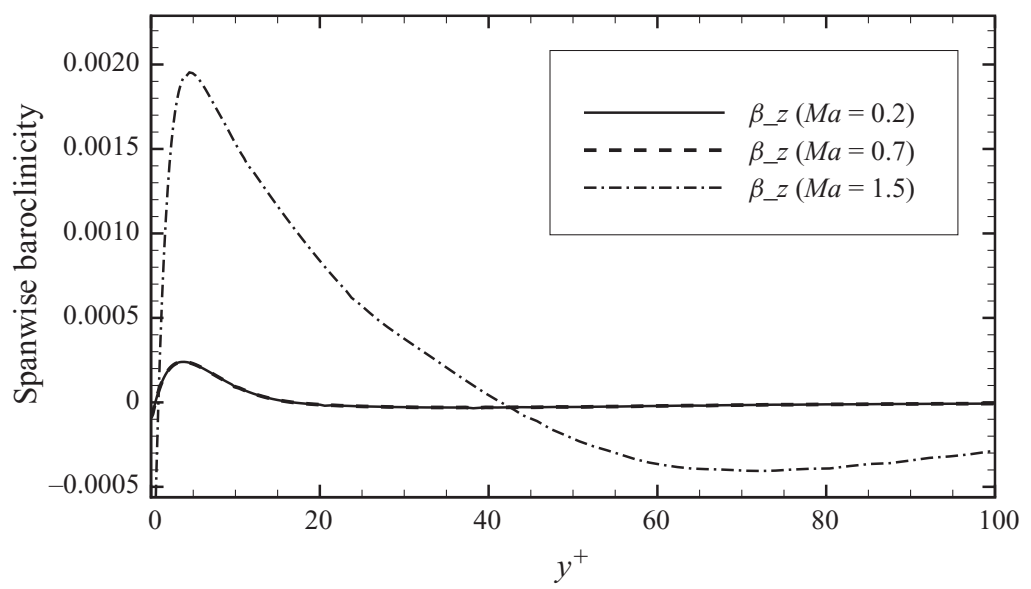

FIGURE 6. Mean spanwise component of the baroclinic vector $\left(\beta_{-} z\right)$ nondimensionalized by the bulk velocity and half channel width close to the wall in wall units.

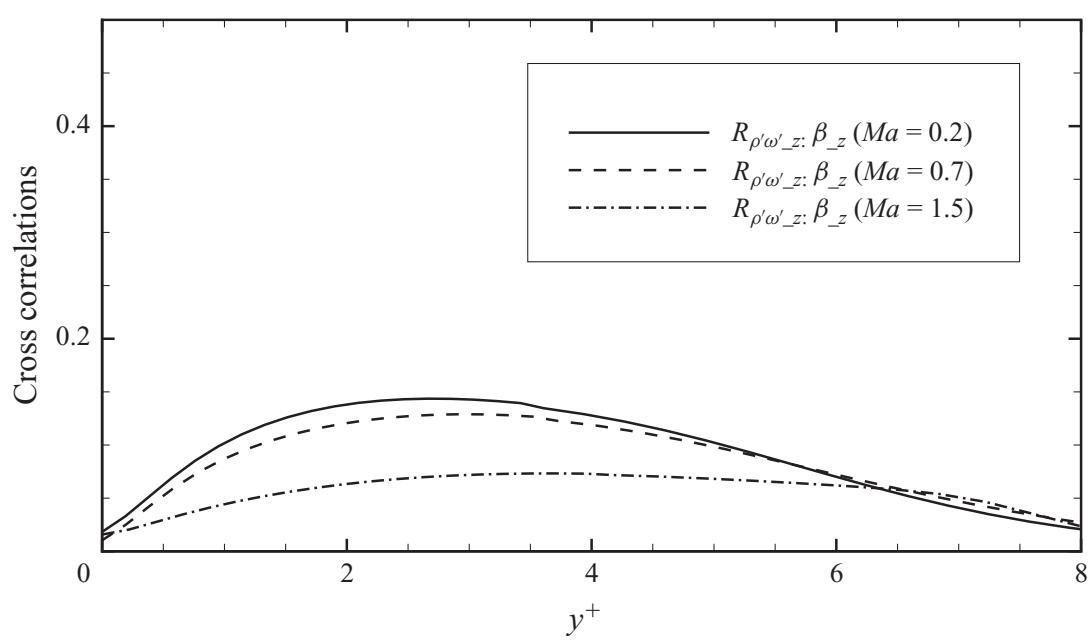

FIgURE 7. Cross-correlation coefficients between $\left\langle\rho^{\prime} \omega_{-}^{\prime} z\right\rangle$ and $\left\langle\beta_{-} z\right\rangle$ close to the wall in wall units.

The terms in (3.6) have been calculated. It is found the magnitude of the first two terms on the right hand of the equation is over 10 times larger than $\left\langle\beta_{3}\right\rangle$ at around $y^{+}=4$. The cross-correlations between $\left\langle\beta_{3}\right\rangle$ and $\left\langle\rho^{\prime} \omega_{3}^{\prime}\right\rangle$ have been generated and shown in figure 7 to examine their relationship very near the wall. Although the magnitudes of the correlation coefficients are not high very close to the wall, peaks in the profiles are located at $y^{+} \approx 3-4$. The peak values decrease with increasing Mach number. One possible reason is that the fluctuating spanwise baroclinic torque term $\left(\left\langle\beta_{3}^{\prime} \beta_{3}^{\prime}\right\rangle\right)$ in the denominator of the correlation definition increases with Mach number. The spanwise baroclinic torque seems to have a closer relationship with the correlation between density and spanwise vorticity at $y^{+} \approx 3-4$ than other locations close to the wall.

To investigate why the peak values of the correlation occur at $y^{+} \approx 4$ instead of on the wall or some other regions, the transport equation for $\left\langle\rho^{\prime} \omega_{i}^{\prime}\right\rangle$ is derived. Through 
Reynolds averaging and some algebraic manipulation of (3.1), it is

$$
\begin{aligned}
\overbrace{\frac{\partial\left\langle\rho^{\prime} \omega_{i}^{\prime}\right\rangle\left\langle u_{j}\right\rangle}{\left.\partial x_{j}\right\rangle}}^{A}=\overbrace{-\frac{\partial\left\langle\rho^{\prime} \omega_{i}^{\prime} u_{j}^{\prime}\right\rangle}{\partial x_{j}}+\left\langle\rho^{\prime} u_{j}^{\prime}\right\rangle\left\langle\omega_{i}\right\rangle}^{D}+\overbrace{\left\langle\rho^{\prime}\left(\omega_{j} \frac{\partial u_{i}}{\partial x_{j}}\right)^{\prime}\right\rangle}^{S} \\
\underbrace{-\left\langle\omega_{i} u_{j}\right\rangle \frac{\partial\langle\rho\rangle}{\partial x_{j}}}_{G} \underbrace{-\left\langle\rho \omega_{i} \frac{\partial u_{j}}{\partial x_{j}}\right\rangle}_{d}+\underbrace{\left\langle\rho^{\prime} \beta_{i}^{\prime}\right\rangle}_{B}+\underbrace{\left\langle\rho^{\prime} \eta_{i}^{\prime}\right\rangle}_{V} .
\end{aligned}
$$

The first term on the left-hand side of (3.7) is the advection term $(A)$, which should be zero. The first term on the right-hand side of (3.7) is the diffusion term $(D)$. The second term $(S)$ denotes the stretching of vorticity. The third term $(G)$ represents the contributions from the gradients of density. The fourth term $(d)$ is the dilatation term. The fifth term $(B)$ is involved with baroclinicity and density fluctuations. The sixth term $(V)$ denotes the influence of viscous effects.

The distribution of the terms in the $\left\langle\rho^{\prime} \omega_{z}^{\prime}\right\rangle$ (or $\left\langle\rho^{\prime} \omega_{3}^{\prime}\right\rangle$ ) equation close to the wall, nondimensionalized by $\rho_{m} U_{m} / h$, for all three cases is shown in figure 8 . The advection term $A$ and the baroclinic term $B$ are essentially zero for all three cases.

For Ma02, shown in figure $8(a)$, the two dominant terms are the dilatation term $d$ and the viscous term $V$. One might wonder why the term $d$ is dominant as the local mean dilatation is almost negligible for $\mathrm{Ma} 02$ (see figure 10). The main reason is that the term $d$ also includes the dilatation-related fluctuation terms which clearly must balance the viscous term $V$ simply because all other terms in (3.7) $(D, S$ and $G)$ are found to be nearly zero. The peaks in these curves occur at about $y^{+} \approx 4$.

For Ma07, shown in figure $8(b)$, the dilatation term $d$ follows the trend and magnitudes established at Ma02. However, the viscous term decreases in magnitude at the expense of growth in other terms $(D, S$ and $G)$ in the transport equation. The interesting feature of figure $8(b)$ is the viscous term. It displays the beginning of a transition from a simple decay process from $y^{+} \approx 4$ to $y^{+} \approx 20$ at Ma02 to one where the maximum is shifted farther away from the wall with a concomitant inflexion at $y^{+} \approx 2$.

The trends established at Ma07 are further enhanced in the case of Ma15, shown in figure $8(c)$. The maximum negative value for the dilatation has moved slightly farther away form the wall to $y^{+} \approx 6$. The viscous term now features within $0<y^{+}<40$ a double crossing of the zero line. The vortex stretching term, $S$ now dominates and is assisted by increased diffusion $(D)$ and transportation through density gradient $(G)$.

It is interesting to note that the peak locations of the profiles of $D, S$ and $G$ change little as Mach number increases. Conversely, the viscous term $V$ close to the wall drops significantly when Mach number increases, due to the combined effect of viscosity and shear. The value of $V$ on the wall, however, remains almost constant with Mach number. Note that the wall temperature and its fluctuation remain the same for all three cases, so do the viscosity and its fluctuation on the wall. For all profiles with peaks/minimums, the locations of these peaks/minimums are around $y^{+} \approx 5$, which in some sense suggests this active region around the edge of viscous sublayer where the peaks of the correlation between the density and the spanwise vorticity are located.

\subsection{Cross-correlations between pressure gradient and vorticity fluxes}

The cross-correlations between pressure gradients and vorticity fluxes have not been previously investigated. $R_{\partial p / \partial x_{k}: \partial \mu \omega_{i} / \partial x_{j}}$ and $R_{\partial p / \partial x_{k}: \partial \omega_{i} / \partial x_{j}}$ for three cases (Ma02, Ma07 

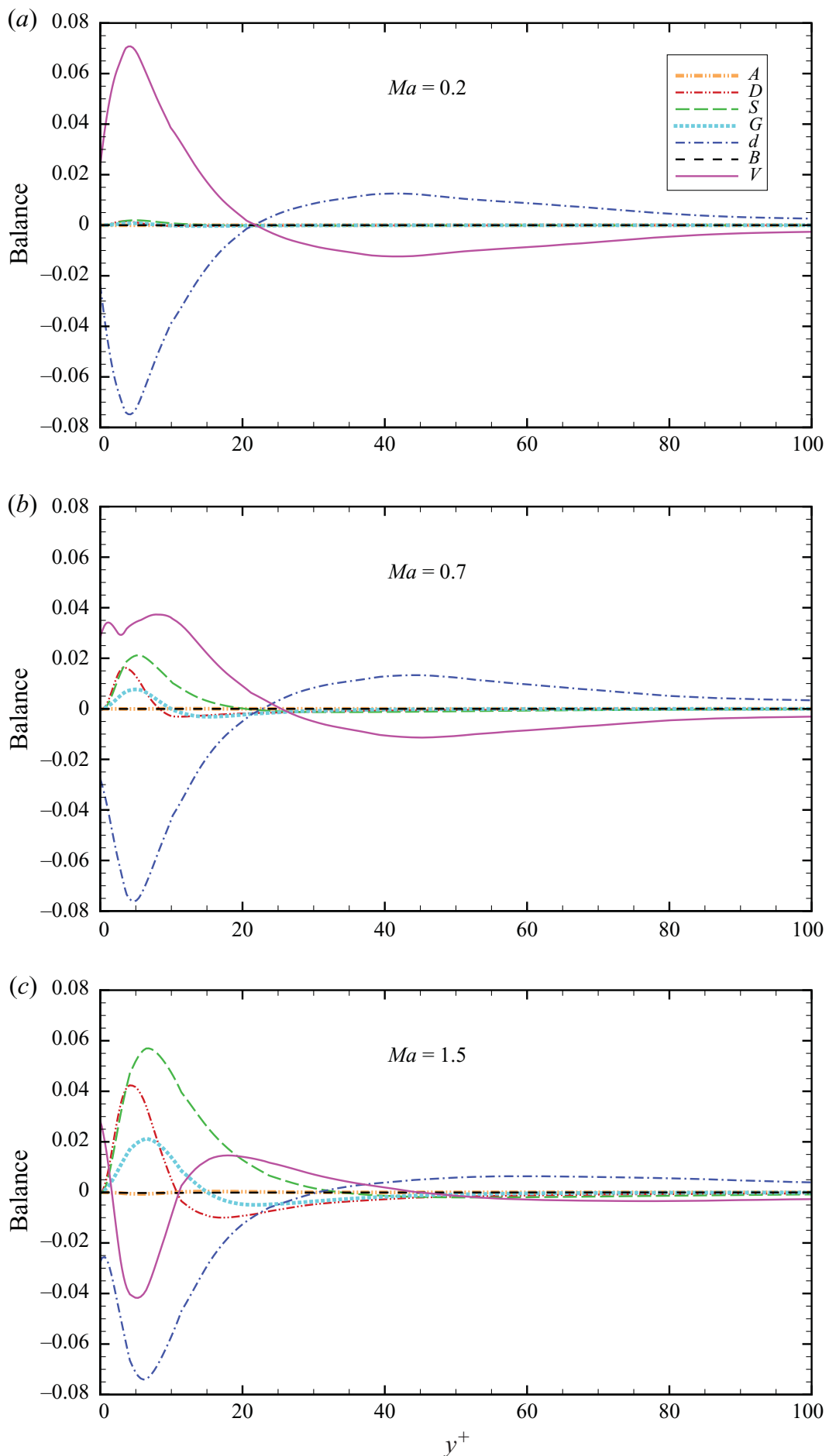

Figure 8. (Colour online) Balance of $\rho^{\prime} \omega_{i}^{\prime}$ (nondimensionalized by $\left.\rho_{m} U_{m} / h\right):(a) M a=0.2 ;(b)$ $M a=0.7$; (c) $M a=1.5$. A denotes $\partial\left\langle\rho^{\prime} \omega_{i}^{\prime}\right\rangle\left\langle u_{j}\right\rangle / \partial x_{j} ; D$ denotes $-\partial\left(\left\langle\rho^{\prime} \omega_{i}^{\prime} u_{j}^{\prime}\right\rangle+\left\langle\rho^{\prime} u_{j}^{\prime}\right\rangle\left\langle\omega_{i}\right\rangle\right) / \partial x_{j}$; $S$ denotes $\left\langle\rho^{\prime}\left(\omega_{j} \partial u_{i} / \partial x_{j}\right)^{\prime}\right\rangle ; G$ denotes $-\left\langle\omega_{i} u_{j}\right\rangle \partial\langle\rho\rangle / \partial x_{j} ; d$ denotes $-\left\langle\rho \omega_{i} \partial u_{j} / \partial x_{j}\right\rangle ; B$ denotes $\left\langle\rho^{\prime} \beta_{i}^{\prime}\right\rangle$ and $V$ denotes $\left\langle\rho^{\prime} \eta_{i}^{\prime}\right\rangle$; where $i=3$ and $j=1,2,3$. 


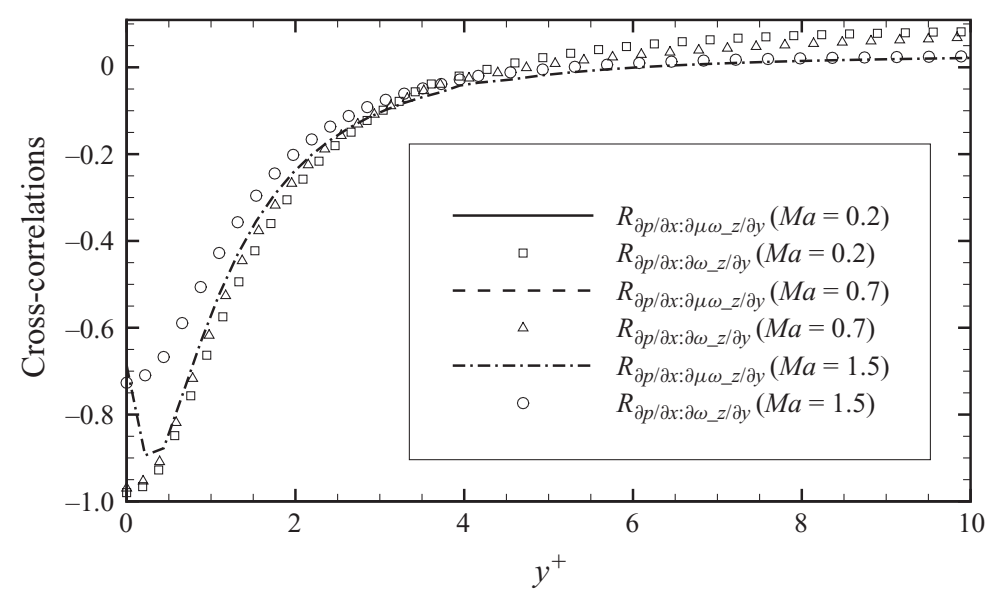

FIGURE 9. Cross-correlation coefficients between streamwise pressure gradient and spanwise vorticity fluxes in the wall-normal direction including the influence of viscosity.

and Ma15) were generated to investigate the influence of Mach number, viscosity, and compressibility/dilatation. Of the 54 correlations considered in $R_{\partial p / \partial x_{k}: \partial \mu \omega_{i} / \partial x_{j}}$ and $R_{\partial p / \partial x_{k}: \partial \omega_{i} / \partial x_{j}}$, only 6 were found to be significant. Additionally, cross-correlations $R_{p: \partial \omega_{i} / \partial x_{j}}$ and $R_{\partial p / \partial x_{k}: \omega_{i}}$ were also generated; however, all these correlations were found to be very small and thus are not shown here. Therefore, only the highly correlated terms within $R_{\partial p / \partial x_{k}: \partial \mu \omega_{i} / \partial x_{j}}$ and $R_{\partial p / \partial x_{k}: \partial \omega_{i} / \partial x_{j}}$ are discussed in the following sections.

The cross-correlation coefficients between streamwise pressure gradient and spanwise vorticity flux in the wall-normal direction $R_{\partial p / \partial x: \partial \mu \omega_{z} / \partial y}$ and $R_{\partial p / \partial x: \partial \omega_{z} / \partial y}$ are shown in figure 9 . The correlation coefficients are high in the magnitude very close to the wall, but negative in sign, which is suggested by (2.5) and (2.8). The absolute value of the correlation coefficients decreases as Mach number increases because of the influence of $\rho f_{x}$ in which the density fluctuation increases with increasing Mach number (see figure 3). The profile of $R_{\partial p / \partial x: \partial \mu \omega_{z} / \partial y}$ for three cases is not very sensitive to Mach number in the region $0.5<y^{+}<5$. The difference between two correlations $R_{\partial p / \partial x: \partial \mu \omega_{z} / \partial y}$ and $R_{\partial p / \partial x: \partial \omega_{z} / \partial y}$ on the wall is negligible except for $0<y^{+}<4$, where there is a clear departure of $R_{\partial p / \partial x: \partial \omega_{z} / \partial y}$ from its viscosity-related correlation for the case Ma15 and it is to this that attention is now turned.

The fluctuations of temperature and viscosity, based on the Sutherland's law (Schlichting 1979), are zero on the isothermal wall and the wall-normal gradients of the temperature and viscosity fluctuations on the wall are very small for the cases considered here, as indicated in figure 3 . The direct influence of the viscosity on the correlation $R_{\partial p / \partial x: \partial \mu \omega_{z} / \partial y}$ is almost negligible on the wall for the current cases. The negligible difference between $R_{\partial p / \partial x: \partial \mu \omega_{z} / \partial y}$ and $R_{\partial p / \partial x: \partial \omega_{z} / \partial y}$ on the wall confirms the weak influence of the viscosity there. Note that the difference will probably not be negligible if the Mach number is much higher than the current cases. Very close to the wall, however, the fluctuations of temperature and viscosity as well as their wall-normal gradients increase significantly as Mach number increases from subsonic to supersonic, which is the main reason for the difference of the two profiles very close to the wall for the case Ma15.

The variation in the mean dilatation $\Theta$ with distance from the wall is presented in figure 10. It is zero on the wall for all three cases and it is negligible everywhere for the case $\mathrm{Ma} 02$. The maximum compression occurs at $y^{+} \approx 7$ and $y^{+} \approx 9$ for the 


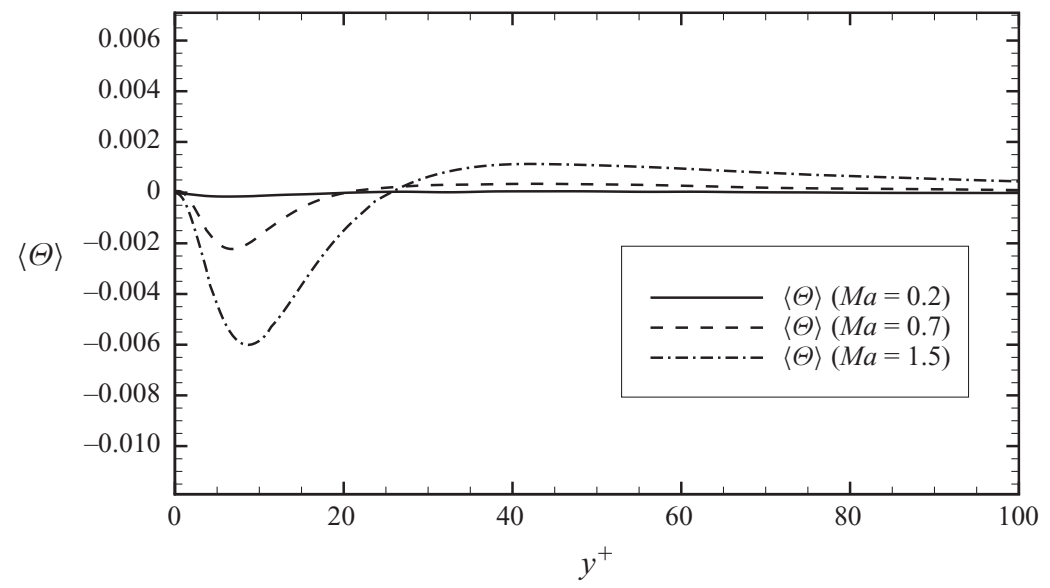

FIGURE 10. The mean dilatation $\Theta$, scaled by $U_{m} / h$.

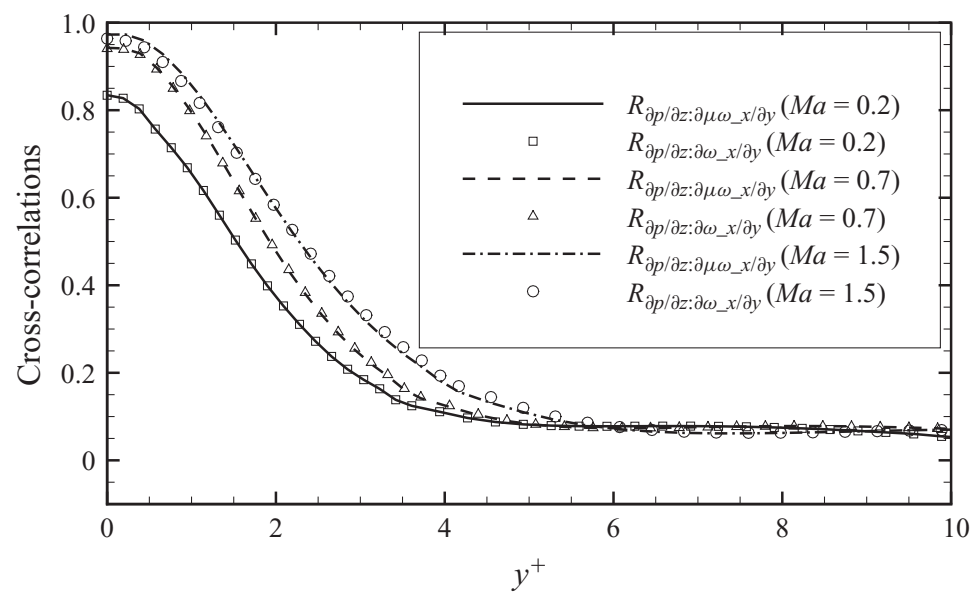

FiguRE 11. Cross-correlation coefficients between spanwise pressure gradient and streamwise vorticity fluxes in the wall-normal direction including the influence of viscosity.

case $\mathrm{Ma} 07$ and Ma15, respectively. The region $y^{+}>30$ is where expansion occurs. The compressibility effect increases with increasing Mach number, but the absolute values remain small for the current cases. The influence of the streamwise gradient of dilatation $\partial \Theta / \partial x$ on the correlation $R_{\partial p / \partial x: \partial \mu \omega_{z} / \partial y}$ seems insignificant.

The cross-correlation coefficients between spanwise pressure gradient and streamwise vorticity flux in the wall-normal direction $R_{\partial p / \partial z: \partial \mu \omega_{x} / \partial y}$ and $R_{\partial p / \partial z: \partial \omega_{x} / \partial y}$ are presented in figure 11. High positive correlation coefficients are observed close to the wall. Again, the relationship is inferred from (2.7) and (2.10). Unlike figure 9, the absolute values of correlation coefficients in figure 11 approach 1.0 as the Mach number increases. The profile of $R_{\partial p / \partial z: \partial \mu \omega_{x} / \partial y}$ for three cases is sensitive to Mach number very close to the wall. It seems that the term $\partial \mu \Theta / \partial z$, compared with the terms $\partial p / \partial z$ and $\partial \mu \omega_{x} / \partial y$ in (2.10), becomes relatively less important on the wall as Mach number increases. The difference between the correlations $R_{\partial p / \partial z: \partial \mu \omega_{x} / \partial y}$ and $R_{\partial p / \partial z: \partial \omega_{x} / \partial y}$ is almost negligible for the current cases; however, the trend suggests that the difference increases slightly with increasing Mach number. Compared with the 


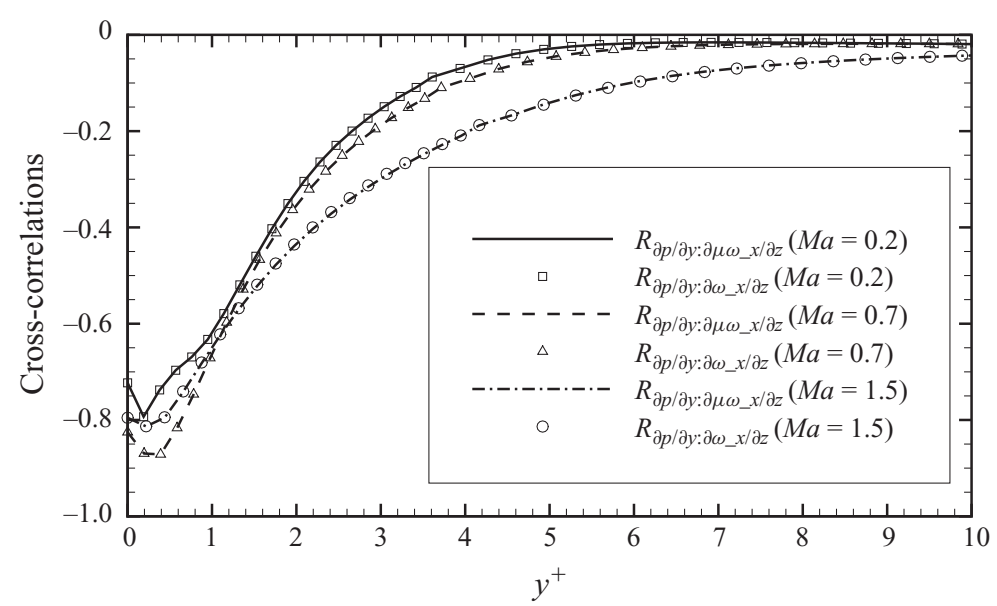

FIGURE 12. Cross-correlation coefficients between wall-normal pressure gradient and streamwise vorticity fluxes in the spanwise direction including the influence of viscosity.

difference for the case $M a=1.5$ observed in figure 9, the difference for the $M a=1.5$ in figure 11 is negligible and this is because $\partial w / \partial y<\partial u / \partial y$ very near the wall.

The cross-correlation coefficients between wall-normal pressure gradient and streamwise vorticity flux in the spanwise direction $R_{\partial p / \partial y: \partial \mu \omega_{x} / \partial z}$ and $R_{\partial p / \partial y: \partial \omega_{x} / \partial z}$ are given in figure 12. It is evident that there is little difference between these correlations. This is obvious on the wall because the term $\partial \mu \omega_{x} / \partial z$ in (2.9) is equal to $\mu \partial \omega_{x} / \partial z$ in (2.6). For the region away from the wall, it indicates that $\partial p / \partial y$ is not well correlated with $\mu$ or $\partial \mu / \partial z$ as $\partial \mu \omega_{x} / \partial z=\mu \partial \omega_{x} / \partial z+\omega_{x} \partial \mu / \partial z$. The correlation coefficients for all three cases are also high on the wall. The profile of $R_{\partial p / \partial y: \partial \mu \omega_{x} / \partial z}$ for three cases does not collapse with Mach number very close to wall. However, it is interesting to note that the highest correlation occurs for the case Ma07 and the profile for the case Ma15 in the region around the edge of viscous sublayer is higher than the other two cases, which could be due to the combined effects of wall-normal gradients of viscosity, dilatation and advection. The wall-normal pressure gradient correlation shows a more complicated behaviour than the wall-tangential pressure gradient correlations.

Equations (2.6) and (2.9) suggest that the wall-normal pressure gradient would possibly be correlated with spanwise vorticity fluxes in the streamwise direction $\left(\partial \mu \omega_{z} / \partial x\right)$. The results from the current simulations demonstrate that the crosscorrelation coefficients for all three cases are positive but below 0.2 on the wall because $\partial \mu \omega_{z} / \partial x$ is much smaller than $\partial \mu \omega_{x} / \partial z$ there. The driving force in the streamwise direction leads to large streamwise shear stress close to the wall, which causes streaks and vortex lines close to the wall to be stretched in the streamwise direction. This streamwise stretching makes streamwise gradients smaller than spanwise gradients.

It is also interesting to note from the above figures that high correlations between pressure gradients and vorticity fluxes only exist very close to the wall. The correlations are almost negligible in the region removed from the viscous sublayer. As (2.4) suggests, the vorticity flux is also affected by advection, besides viscosity and pressure gradients. The advection is negligible very close to the wall and viscosity effects dominate; however, advection begins to play a dominant role away from the wall. Advection is an important reason that the high correlation between pressure gradient and vorticity flux decreases with distance from the wall. In other words, when the vorticity is generated on the wall, the pressure gradient plays an important role as 
advection very near the wall is small. The vorticity is then diffused into the flow first due to viscosity before being advected away.

\section{Summary}

DNS of three isothermal-wall-bounded turbulent channel flows (cases Ma02, Ma07 and Ma15) have been carried out with $R e \approx 2800$ using DGM. The interactions among pressure, density, vorticity and their gradients have been investigated. A summary of the current findings is as follows.

The correlations among pressure, density and vorticity are generated and studied. Pressure and density are perfectly correlated on the wall, but correlations between pressure and vorticity components are negligible. Among the correlations between density and vorticity components, it is found that a high correlation between density and spanwise vorticity occurs at $y^{+} \approx 4$, where the peak mean spanwise baroclinicity is located. The relationship between the spanwise baroclinicity and the correlation was analysed. The difference between the evolution of density and spanwise vorticity very near the wall is discussed and temperature has a great effect on the distribution of near-wall density streaks. The balance of $\left\langle\rho^{\prime} \omega_{z}^{\prime}\right\rangle$ and related influential terms were formulated and analysed. It is found that the peaks/minimums of the profiles are located at around $y^{+} \approx 5$.

The connection between the pressure gradient and vorticity flux for compressible turbulent flow with variable viscosity was derived, which further extends the vorticity dynamics theory based on Lighthill's essays (1963) in laminar boundary layers. Correlations were employed to evaluate their linkage for the first time. The correlations between streamwise pressure gradient and spanwise vorticity flux in the wall-normal direction, between spanwise pressure gradient and streamwise vorticity flux in the wall-normal direction and the correlation between wall-normal pressure gradient and streamwise vorticity gradient in the spanwise direction, are very high $(0.7-1.0)$ very close to the wall. It is also found that both Mach number and viscosity affect the correlation between streamwise pressure gradient and spanwise vorticity flux in the wall-normal direction very close to the wall.

The authors would like to thank Dr G. Karniadakis and his CRUNCH group and $\operatorname{Dr}$ M. Kirby for providing the original discontinuous Galerkin code and the related helpful email discussions. The reviewers are acknowledged for their insightful comments. One reviewer is especially thanked for suggesting a derivation of the spanwise baroclinic torque as a function of the correlation between density and spanwise vorticity. The research was funded through grants from NSERC Canada. Computing resources were provided by HPCVL (www.hpcvl.org).

\section{REFERENCES}

Andreopoulos, J. \& Agui, J. H. 1996 Wall-vorticity flux dynamics in a two-dimensional turbulent boundary layer. J. Fluid Mech. 309, 45-84.

Cockburn, B., Karniadakis, G. E. \& Shu, C.-W. 2000 Discontinuous Galerkin Methods - Theory, Computation and Applications. Springer.

Coleman, G. N., Kim, J. \& Moser, R. D. 1995 A numerical study of turbulent supersonic isothermal-wall channel flow. J. Fluid Mech. 305, 159-183.

GAD-El-HaK, M. 1990 Control of low-speed airfoil aerodynamics. AIAA J. 28 (9), 1537-1552.

Karniadakis, G. E. \& Sherwin, S. 2005 Spectral/hp Element Methods for Computational Fluid Dynamics, 2nd edn. Oxford Science Publications. 
Koumoutsakos, P. 1999 Vorticity flux control for a turbulent channel flow. Phys. Fluids 11 (2), 248-250.

LEe, C. \& Kim, J. 2002 Control of viscous sublayer for drag reduction. Phys. Fluids 14 (7), 2523-2529.

Lighthill, M. J. 1963 Introduction. Boundary Layer Theory. In Laminar Boundary Layers, chap. II Oxford University Press.

Moser, R. D., KIm, J. \& Mansour, N. N. 1999 Direct numerical simulation of turbulent channel flow up to $R e_{\tau} \approx 590$. Phys. Fluids 11 (4), 943-945.

PANTON, R. L. 1984 Incompressible Flows. Wiley Interscience.

Schlichting, H. 1979 Boundary-Layer Theory, 7th edn. McGraw-Hill Book Company.

WEI, L. 2009 Direct numerical simulation of compressible and incompressible wall bounded turbulent flows with pressure gradients. PhD thesis, Queen's University, Kingston, ON, Canada.

Wei, L. \& Pollard, A. 2010 Direct numerical simulation of compressible turbulent channel flows using discontinuous Galerkin method. Comput. Fluids (Tentatively accepted).

WU, J. Z. \& WU, J. M. 1998 Boundary vorticity dynamics since Lighthill's 1963 article: review and development. Theor. Comput. Fluid Dyn. 10, 459-474.

WU, J. Z., WU, J. M. \& WU, C. J. 1988 A viscous compressible flow theory on the interaction between moving bodies and flow field in the $(\omega, \vartheta)$ framework. Fluid Dyn. Res. 3, 203-208.

Wu, J. Z., Wu, X. H. \& Wu, J. M. 1993 Streaming vorticity flux from oscillating walls with finite amplitude. Phys. Fluids A 5, 1933-1938. 